\title{
Las teorías implícitas en el conocimiento social $(*)$
}

\section{M.a José Rodrigo(**)}

Universidad de La Laguna

Nuestras categorias del mundo fisico son relativamente objetivas y con escasas diferencias interindividuales, dado que corresponden a conglomerados de atributos de nuestra experiencia sensorial inmediata. No obstante, nuestras representaciones del mundo social están menos guiadas por los datos sensoriales inmediatos y depende más de propiedades y sucesos de carácter inferencial (estatus, inteligencia, agresividad, tristeza, etc.). Para entender y planificar nuestro comportamiento en el mundo social llegamos a elaborar marcos interpretativos relativamente idiosincrásicos que podrían considerarse como verdaderas teorías espontáneas o implícitas. Por ejemplo, aquellos que comparten la teoría de los grupos marginales (alcohólicos, drogadictos, etc.), como «enfermos» sin duda perciben e interpretan los comportamientos de estos grupos de modo muy diferente a los que mantienen la teoría de la marginalidad como «vicio».

Las teorias implicitas son unidades representacionales complejas que incluyen multitud de proposiciones organizadas en torno a un dominio concreto del mundo social. Sus funciones son múltiples; permiten interpretar o explicar comportamientos, establecer predicciones y tienen un valor prescriptivo marcando pautas o directrices a nuestra propia conducta social.

El objetivo de este artículo es realizar un acercamiento al tema, conjugando dos líneas de investigación de origen dispar. En primer lugar, las aportaciones de la psicología social europea, que precisamente ha acuñado la denominación de "teoría implícita» (Forgas, 198r; Goodnow, 1981; Wegner y Vallacher, 1977, 1981).

Esta orientación resalta la naturaleza esencialmente social del conocimiento en el hombre, siguiendo tradiciones teóricas tanto de la psicología como de la sociología: Wundt, James, Mead, Durkheim, Weber y otros. Consecuentemente, las teorías se conciben como productos de todo un trasfondo colectivo de influencias.

La segunda orientación que tomaremos en cuenta corresponde a la psicología cognitiva, específicamente las denominadas teorías de esquemas, que abordan a su modo el tema de las representaciones en el conocimiento social.

(*) La presente investigación se ha desarrollado bajo el patrocinio de la CAICYT (Proyecto núm. I695-82), de cuyo equipo investigador forma parte la autora.

${ }^{* *}$ ) Dirección de la autora: Universidad de La Laguna, Facultad de Filosofia y Letras. Dpto. de Psicología Evolutiva. La Laguna, Tenerife. 
Las últimas posiciones críticas de los teóricos del procesamiento de la información a la metáfora del ordenador, han acercado sus planteamientos a los de la psicología social europea (Seoane, 1982; Garzon, 1984). Sin embargo, su distinta formación les ha llevado a insistir en el estudio de los procesos cognitivos que utiliza el individuo para procesar la información social, tanto desde una perspectiva evolutiva (Shantz, 1975; Flavell y Ross, 1981; Lamb y Sherrod, 1981), como desde la perspectiva de los adultos (Higgins, Herman y Zanna, i98 I). Concretamente, basándose en el concepto de esquema formulado originalmente por Bartlett (1932) y Piaget (1926) y posteriormente, recogido por la Inteligencia Artificial (Schank y Abelson, 1977; Abelson, 1975, 1981), han analizado detalladamente las propiedades estructurales y funcionales de algunas unidades representacionales de nuestro sistema de conocimiento como las categorías sociales, los guiones (scripts o esquemas de eventos), etc.

En suma, tanto la psicología social como la psicología cognitiva, han confluido actualmente en el estudio del conocimiento social. Sus perspectivas son complementarias: los psicólogos sociales insisten en la importancia del contexto sociocultural en la conformación del conocimiento. Asimismo, los factores motivacionales, afectivos y normativos, dependientes del contexto, inciden sobre el conocimiento social, de modo que éste no es el producto de un procesamiento neutro y formal. En contraste, los psicólogos cognitivos han analizado con más detalle las propiedades estructurales y de procesamiento de algunas unidades representacionales del conocimiento social y su relación con la memoria, percepción, procesos de comprensión, etc.

En nuestra opinión, se requieren ambas perspectivas para poder dar una imagen más completa de lo que son las teorias implícitas. Abundar sólo en su origen social, no contribuye a explicitar su naturaleza estructural y funcional mientras que insistir sólo en esta última, limita y empobrece nuestras concepciones sobre la génesis del conocimiento. Las teorías implícitas son el producto de una delicada interacción entre estrategias individuales de procesamiento de información y procesos socioculturales a gran escala.

\section{A) TEORIAS CIENTIFICAS VERSUS TEORIAS INTUITIVAS}

El conjunto de conocimientos desarrollados por el hombre de la calle en su intento de comprender la realidad social, poder anticipar el futuro, y planificar su comportamiento, se articula en varios tipos de unidades representacionales, una de las cuales son las llamadas «teorías».

Wegner y Vallacher (1977) sugieren que existe una considerable organización y regularidad en la estructuración de los paquetes informacionales que conforman las teorías intuitivas. Por ello, siguiendo la analogía del científico con el lego, podemos sostener que existe un cierto parecido formal entre las teorías científicas y las que elabora el hombre de la calle (De Vega, 1983).

Tanto las teorías científicas como las intuitivas están constituidas por un conjunto de conceptos y de eslabones que establecen relaciones entre éstos. Asimismo, ambas comparten funciones interpretativas; una 
vez reunidos los datos se elaboran explicaciones causales basadas en los postulados teóricos; o bien permiten establecer predicciones sobre sucesos futuros; por último, incluyen «rutinas .operarivas» sobre el modo correcto de actuar.

Las diferencias fundamentales entre las teorias del cientifico formal y las del lego estriban en su grado de exactitud y de accesibilidad subjetivas.

El hombre de la calle se siente personalmente inclinado a considerar correctas sus creencias sin necesidad de someterlas a prueba. Por el contrario, los científicos formales reconocen que sus teorias tienen un carácter provisional y deben ser constantemente sometidas a verificaciones empiricas. No obstante, habria que matizar esta última afirmación. Los cientificos formales suelen también considerar correctas sus predicciones (el llamado sesgo confirmatorio, Tweney, Doherty y Mynatt, 1981). Pero debido a su entrenamiento en el método cientifico, se atienen a las normas y prescripciones de la ciencia para justificar sus afirmaciones: representatividad de la muestra, verificabilidad de las proposiciones, falseabilidad de los resultados, etc. En este sentido puede decirse que el entrenamiento en el método científico corrige las tendencias confirmatorias de los científicos formales. Las teorias intuitivas no sufren ese proceso corrector (al menos no de modo sistemático), por tanto pueden persistir en la mente de los sujetos concepciones totalmente erróneas.

En cuanto a la accesibilidad subjetiva de las teorias, el cientifico ostenta una posición ventajosa respecto al lego ya que, al aceptar las convenciones sociales de la ciencia, constantemente se ve forzado a hacer explícitas las proposiciones de su teoría. Por el contrario, como se verá en páginas posteriores, el hombre de la calle sólo excepcionalmente las hace explícitas.

Se han realizado numerosas investigaciones para analizar los sesgos que provocan las inexactitudes y errores en las teorías del cientifico intuitivo. A continuación expondremos algunos de ellos, agrupándolos en función de las tareas que lleva a cabo el científico intuitivo para elaborar una teoría, siguiendo los mismos pasos del científico formal (Ross, $19^{81}$ ).

\section{Errores en la caracterización de los datos y la muestra}

En general, las personas son bastantes eficaces en estimar frecuencias, proporciones o porcentajes, esto es, en caracterizar una muestra. Sin embargo, esto sólo es cierto cuando los datos de la muestra son accesibles en el momento; discretos y enumerables y no están muy dispersos temporalmente (Nisbett et al., 1983). En el medio social en el que se mueve el individuo rara vez se presentan los datos de esta manera. Lo frecuente es que sean poco accesibles, entremezclados con datos irrelevantes, poco enumerables y temporalmente dispersos. En este caso, los procesos de recuperación se dificultan extraordinariamente, y las posibilidades de error se incrementan (Peterson y Beach, 1967). En esta circunstancia, los sujetos utilizan un procedimiento que simplifica la labor de "recogida de datos»: acuden únicamente a los fenómenos más salientes (los más atractivos, o que están ligados 
emocionalmente a experiencias pasadas, etc.), que son más evocables en su memoria (Kahneman y Tversky, 1973; Rothbart et al., 1978; Taylor y Fiske, 1978).

\section{Sesgos en la generalización de la muestra}

La mayor parte de los juicios sociales requieren que el individuo haga una generalización inductiva para inferir parámetros de una población, desde la información recogida de una muestra. Las investigaciones demuestran que las personas no valoran suficientemente la importancia del tamaño de la muestra para realizar juicios representativos de la población general (Borgida y Nisbett, 1977). Asimismo, la homogeneidad de la muestra facilita la generalización a partir de casos aislados (Nisbett et al., $19^{83}$ ).

\section{Sesgos en las evaluaciones atribucionales}

Una vez recogida la muestra de datos, las personas empiezan a generar relaciones causales para conectarlos. Para ello utilizan el siguiente principio: los efectos son asignados a las causas con las que covarian. Este procedimiento, aunque suele tener éxito, no resulta siempre infalible. Existen datos que, aunque confluyen en el espacio y en el tiempo, no guardan ninguna relación causal. En general, no se da un análisis objetivo de los hechos reales sino que son las expectativas sobre los resultados las que sirven de guía para agrupar y relacionar causalmente los hechos. Una vez que se ha descubierto una explicación satisfactoria para un fenómeno, cesa inmediatamente la búsqueda de nuevas causas que pueden resultar igualmente relevantes, según un principio de parsimonia o de economía explicativa (Nisbett y Ross, 1980; Schustack y Sternberg, 1981).

Por último, las diferencias en la percepción de sucesos entre los actores y los observadores determinan una serie de sesgos atribucionales. Asi, los sujetos tienden a exagerar el grado de consistencia entre su conducta y los resultados de ésta (ilusión de control, Langer, 1975; Alloy y Seligman, 1979; Alloy y Tabachnik, 1984). Los observadores, por su parte, fracasan al realizar atribuciones de los actores porque subestiman el impacto de los determinantes situacionales y sobreestiman el carácter disposicional de las acciones (error fundamental de atribución, Anderson y Ross, 1980; Nisbett y Ross, 1980; Ross, 1977).

\section{Errores en la predicción}

A la hora de llevar a cabo una predicción, los errores cometidos en la selección de la muestra pesan extraordinariamente. Así, la dificultad de contar con datos de tipo estadístico que reflejen más correctamente la realidad o la incapacidad de realizar cómputos intuitivos a partir de los datos disponibles, lleva a los sujetos a basarse en estrategias de tipo heurístico. Estas actúan como poderosas guías para la elaboración de los juicios predictivos. En particular, los heurísticos de representatividad (un suceso es probable si la evidencia disponible es representativa 
del modelo mental de ese suceso) y accesibilidad (un suceso es probable en la medida en que se puede recuperar y representar con vividez en nuestra memoria) han sido ampliamente estudiados (Kahneman y Tversky, 1972, 1973; Kahneman, Slovic y Tversky, 1982).

\section{Errores en la comprobación y revisión de las teorias}

Las evidencias que no concuerdan con las predicciones del científico intuitivo no actúan de revulsivo para modificar la teoría. Por el contratio, el científico intuitivo tiende a perseverar en las creencias establecidas, aunque la nueva información demuestre su inoperancia (Ross y Lepper, 1980). Los estudios realizados con niños (KarmiloffSmith e Inhelder, 1974), han descubierto la misma tendencia confirmatoria. Los niños tienden a enfatizar la evidencia favorable a sus hipótesis descuidando los datos desfavorables.

La descripción de las inexactitudes y errores que comete el hombre de la calle al elaborar sus teorías sobre la realidad social, responde a un criterio de contrastación de estas concepciones intuitivas con el producto elaborado de una teoría formal. Por el contrario, si juzgamos la "calidad» de esas teorías espontáneas en relación a su finalidad pragmática y eficacia adaptativa, la valoración es mucho más positiva. Dada la complejidad y ambigüedad de los datos en los contextos sociales y la urgencia de planificar respuestas, resulta un acierto la utilización de procedimientos poco exhautivos pero más económicos y rápidos para procesar todo ese tipo de informaciones (De Vega, 1984).

\section{B) LA DIMENSION IMPLICITO-EXPLICITO EN LAS TEORIAS INTUITIVAS}

La denominación de teorias implícitas hace referencia al carácter inaccesible de las proposiciones que constituyen las teorías. Concretamente, el hecho de que el individuo entienda los aspectos del mundo social a través de sus teorías, y no por el análisis de la teoría en sí misma, es lo que hace que sea caracterizada como implícita en su operación (Polansky, 1969; Wegner; en prensa).

En general, la mayor parte de las teorías intuitivas se consideran como implícitas. Sirvan de ejemplos los estudios sobre las teorías implícitas de la personalidad (Bruner y Tagiuri, 1954; Rosenberg y Sedlak, 1972; Schneider, 1973, entre otros) y sobre las teorías implícitas que sostienen los niños acerca de sus semejantes, en función de la edad, sexo e inteligencia verbal (Hones, 1979). En ambos casos, el sujeto «ve» el mundo a través de su teoria y no es consciente de su propio sistema de filtros.

Por el contrario, la denominación de teorías explícitas hace referencia al carácter accesible de ciertas creencias a la conciencia del individuo. Cuando éste elabora explicaciones, descripciones, hipótesis y atribuciones sobre un dominio del mundo social, se trata de una teoria explícita.

El análisis de las teorías explícitas de las personas, sobre una variedad de fenómenos, es bastante frecuente en la actualidad. Son 
ejemplos los estudios pioneros de Heider (1958) sobre las atribuciones y las descripciones del lenguaje común sobre la personalidad de los otros (Bromley, 1977). Por otra parte, la sabiduría popular o el sentido común incluyen un conjunto de máximas, proverbios y refranes que son manifestaciones populares de teorias explícitas.

¿Qué conexiones existen entre las teorías implícitas y explícitas? Aunque no hay correspondencia directa y unívoca entre nuestro pensamiento explícito y la teoría implícita subyacente (éste es el principal problema de los datos verbales obtenidos con el método introspectivo), se ha comprobado que existe una cierta interrelación entre ambos tipos de teorías (Wegner y Vallacher, 1981). Concretamente, existen algunos factores que facilitan la transformación de una teoría implícita en explícita.

Existen factores psicológicos que pueden vencer la natural resistencia de las teorías implícitas a nuestros intentos introspectivos. En primer lugar, la recencia de las observaciones sobre los efectos de una teoría implícita cuando opera (Ericcson y Simon, 1980; White, 1980). El grado de recencia y saliencia cognitiva de la aplicación particular de una teoria determina en qué medịda el sujeto puede hacerla explícita. En segundo lugar, el carácter planificado y selectivo de las autoobservaciones favorece la accesibilidad a la teoría. En situaciones experimentales se puede recomendar a los sujetos que dirijan la atención sistemáticamente hacia una determinada teoría haciéndola explícita. Por último, el hecho de que la teoría implícita no sea confirmada de modo patente. Quizá sea este último aspecto el que requiera mayor aclaración. Las personas se ven más inclinadas a hacer explícitas sus teorías en relación a las nuevas experiencias del individuo. Por el contrario, las teorias implícitas que son confirmadas repetidamente, ya sea como resultado de la aplicación del sesgo confirmatorio o como resultado de su tendencia a producir los efectos que predice (profecía autocumplida), son las menos accesibles y producen menores evidencias de su actuación.

También hay factores sociales determinantes de la transformación de las teorias implícitas en explícitas. En primer lugar, la disponibilidad de términos descriptivos pertenecientes al léxico común para expresar la teoría. En segundo lugar, el grado en que la presión social promueve la atención hacia las teorías implícitas. Por ejemplo, la mera presencia de una audiencia, que destaque el protagonismo de una persona induce en ésta un análisis más exhaustivo de sus propias creencias y centra en ellas su foco de atención (Duval y Wicklund, 1972). Un último factor es la valoración social de las teorias implícitas, puesto que los seres humanos pueden negar la articulación explícita de éstas cuando se juzgan irracionales, inmorales o impropias (Snyder y Wicklund, en prensa).

Consideremos ahora la cuestión inversa, es decir, qué factores 'determinan el grado en que una teoría explícita puede influir sobre una teoría implícita. ¿En qué medida el entrenamiento y la adquisición de conocimientos sobre un determinado campo determina la transformación de las teorías implícitas correspondientes?

En principio, el conocimiento explícito de las propias capacidades conduce a, un remodelamiento de nuestras ideas sobre éstas. De la misma manera que los niños manejan mejor sus capacidades mnémicas a medida que conocen los mecanismos de almacenaje y recuperación de

$$
\text { a medida que conocen los mecanismos de almacenaje y recuperación de }
$$


información (metamemoria, Flavell y Wellman, 1977), ‘j personas conocen mejor sus propias limitaciones a través de un conocimiento explícito de éstas.

Otro factor que puede facilitar la transformación de teorias implicitas a partir de conocimientos explícitos es la revisión de nuestros deseos y preferencias. Su conocimiento explícito, ya sea por autoobservación o a partir de informaciones recibidas de los otros, hace que la teoría implícita subyacente se haga evidente y que a su vez sea objeto de revisión (Vallacher, 1980). Por ejemplo, una vez que conocemos nuestro rechazo hacia determinados grupos raciales, podemos poner en tela de juicio la teoría implicita que guia tales actitudes. Si la valoración de esta teoría es negativa, evitaremos en adelante las actitudes y comportamientos racistas. En este sentido, la revisión de teorias implícitas, a partir del conocimiento explícito de sus consecuencias, es la base del autocontrol.

En conclusión, una teoria implícita se puede modificar a través de su representación explícita. Sin embargo, esta afirmación podría hacernos suponer que las personas pueden cambiar sus teorías implicitas cuando se descubre que son ineficaces, inmorales o indeseables. Pero esto no ocurre siempre. Como las teorías son el único medio disponible de imponer orden y significado a la realidad, incluso una teoría desacreditada puede ser preferida a otra más plausible. Como Kuhn (1970) ha sugerido, en relación a las teorias científicas, una teoria desacreditada sólo se abandona cuando se cuenta con una teoría mejor. Las tendencias confirmatorias son tan intensas tanto en el científico como en el hombre de la calle, que de hecho se da una escasa movilidad en la transformación y modificación de teorías.

\section{C) LAS TEORIAS IMPLICITAS COMO ESQUEMAS}

Hemos descrito algunas caracteristicas de las teorias intuitivas: su similitud con las teorías científicas y su grado de accesibilidad a la conciencia de los sujetos. Sin embargo, se echa en falta un análisis más detallado de las propiedades estructurales y funcionales de las teorias y su relación con el conjunto del sistema cognitivo.

Para llevar a cabo esta tarea hemos partido de una hipótesis de trabajo: las teorías son estructural y funcionalmente análogas a esquemas de conocimiento social. Esta hipótesis será sometida a prueba en el siguiente artículo.

La similitud entre las propiedades de los esquemas y las teorias implícitas es justificable por varias razones:

- Las teorías, al igual que los esquemas, representan "paquetes» de conocimiento prototípico que apresan ciertas regularidades en las situaciones, comportamientos, personajes, ideas, que perciben, los sujetos en su entorno. En este sentido, están compuestos de una serie de elementos o proposiciones ordenados según un continuo de tipicidad. Como tendremos ocasión de demostrar en la investigación que se presenta en el siguiente articulo, las proposiciones o conjuntos de ideas que forman parte de una teoria, varian desde las más características y definitorias hasta las menos típicas.

- Las funciones de las teorías implícitas son presumiblemente 
similares a las de los esquemas: ayudan a explicar, predecir y planificar la conducta. Los esquemas proporcionan marcos interpretativos para los fenómenos, guiando, por tanto, los procesos de comprensión. No obstante, su principal papel es contribuir a anticipar ciertos resultados a través de mecanismos inferenciales y completar, así, la información con la que cuenta el sujeto. En este sentido, la activación de esquemas tiene un papel activo en la comprensión (Minsky, 1975; Kuipers, 1975; Rumelhart y Ortony, i 977) y en el recuerdo (Neisser, 1976; Kintsch y Van Dijk, 1978; Schank y Abelson, 1977; Graesser et al., 1979). Por último, es fundamental su papel en la planificación de la conducta. Los esquemas no sólo contienen información conceptual sobre el mundo, sino que tienen un carácter procedimental dirigido a la acción (Piaget, I950; Minsky, I975; Schank y Abelson, 1977).

Siguiendo la analogia de las teorias implicitas con las representaciones esquemáticas, es razonable suponer que aquéllas también tengan un papel relevante en los procesos de comprensión, memoria y planificación de acciones. Sin embargo, dada la práctica ausencia de estudios en este sentido, no podemos ir más allá de la mera suposición.

- Las teorías implicitas al igual que los esquemas se adquieren a partir de las experiencias personales en situaciones recurrentes. Tales recurrencias vienen propiciadas por las normas sociales y la cultura, que tienden a «uniformizar» la posible variedad de contextos y situaciones sociales.

- Las teorias implícitas, al igual que los esquemas, se adquieren a las normas sociales y, en general, por todo el entramado social. Fueron las investigaciones pioneras de Barlett (1932) las que pusieron de manifiesto el efecto de la cultura y la sociedad en el sistema cognitivo de los individuos.

- Las teorías y los esquemaś son representaciones muy versátiles, en principio válidas para todo tipo de dominios de conocimiento. Prácticamente, todos los contenidos de la memoria humana se organizan total o parcialmente en esquemas. De igual modo, las teorias implicitas se aplican a varios dominios de conocimiento. Hasta la fecha, se han desarrollado estudios sobre dominios tales como el autoconcepto (Lepper, Greene y Nisbett, 1973; Wegner y Vallacher, I977), las relaciones sociales (Wish, Deutsch y Kaplan, 1976; Rands y Levinger, 1979), el concepto de anormalidad (Rosenhart, 1973; Chan y Jackson, 1979) y sobre la personalidad (Bruner y Tagiuri, 1954; Schneider, 1973; Wegner, 1977).

\section{D) RASGOS DIFERENCIALES DE LAS TEORIAS IMPLICITAS}

Hemos considerado las teorías como estructuras de conocimiento de tipo esquemático que se elaboran como producto de influencias sociales y culturales.

No obstante, pecaríamos de excesivamente simplistas si nố jlimitáramos a postular una total identidad entre las teorias implicitas/y otras unidades representacionales, sin hacer ningún tipo de precisiones. Veamos las características diferenciales entre las categorias y las teorias implícitas. 
Las categorías son unidades esquemáticas de orden inferior, lo cual supone que:

- Los patrones de covariación a los que së refieren tienen una base fuertemente sensorial.

- Los patrones de acción que determinan son rutinas motrices.

- La categorización admite pocos grados de libertad interindividual. Nuestro aparato psíquico está diseñado para construir categorías realistas, que guarden una correspondencia con las pautas correlacionales del mundo. De ahi las escasas diferencias individuales y transculturales en la construcción y organización de categorias.

- Los procesos inferenciales en la categorización son relativamente escasos (atribución de propiedades no directamente observables) y ocurren de un modo prácticamente automático.

En cambio, las teorías implícitas son unidades representacionales de alto nivel, lo cual supone que:

- Los patrones de covariación no tienen una entidad sensorial inmediata, sino que vienen definidos por la retícula de relaciones interpersonales y de propiedades sociales inferidas.

- Los patrones de acción determinados son planes de orden superior que controlan de modo jerárquico otros planes secundarios y así sucesivamente. Todos ellos se dirigen a metas del mundo social y pueden desarrollarse en escalas temporales de gran magnitud: días, meses y años.

- Dado el carácter inferencial y social de las teorías implícitas, el sustrato cultural y la experiencia personal acumulada determinan teorías implícitas alternativas a veces de orientación muy dispar, aunque versen sobre el mismo dominio.

- Los procesos inferenciales o «guiados conceptualmente» tienen un gran peso tanto en los juicios como en los comportamientos sociales derivados de las teorías.

A modo de ilustración de las peculiaridades de las teorías implíciias, consideremos una teoría hipotética en torno al papel social de la mujer. Una concepción tradicional de la mujer incluye un conjunto de atribuciones caracteriales ("es discreta», "dulce», "callada»), ocupacionales («cuida y busca el agrado del marido») y maternales («es responsable de la crianza de los hijos»). Esa misma concepción origina patrones de comportamiento social a largo plazo muy característicos, en relación con la crianza de los hijos («a las hijas se las educa-para ser futuras esposas»), las relaciones de pareja («el hombre busca una mujer sumisa y escasamente autoasertiva), etc.

Evidentemente, la concepción tradicional de la mujer no es la única alternativa disponible, al menos en nuestra sociedad. Muchos individuos optan por una concepción de la mujer como «personalmente emancipada». Los que comparten esta u otras teorías alternativas sobre la mujer, sostendrán juicios, expectativas y comportamientos muy dispares en torno a ésta.

La causa de ello es que las teorías alternativas en torno a un mismo dominio guían los procesos inferenciales en distinta dirección. Supongamos que en una reunión de amigos los hombres se disponen a hablar de «temas masculinos" mientras las mujeres se retiran para hablar entre 
ellas. Un observador que comparta la concepción tradicional sobre la mujer consideraría el hecho normal y justificaría la segregación de la conversación por sexos. En cambio, un observador que comparta la segunda teoría tendería a juzgar el hecho como una aberración social, manifestación de costumbres patrimonialistas injustificables. Incluso podría especularse que el recuerdo posterior del acontecimiento sería muy distinto en ambos observadores, pues los mecanismos inferenciales determinan procesos abstractivos y reconstructivos de la información (Spiro, 1980; Kintsch y Van Dijk, 1978).

El campo de las teorías implícitas apenas ha recibido un tratamiento sistemático por parte de los psicólogos, pero sus posibilidades son patentes. Las grandes diferencias entre los grupos humanos y entre los individuos en su percepción, juicios, actitudes, y comportamientos sociales son fenómenos ampliamente constatados por la psicología. Con la noción de teorías implícitas se abre un nuevo panorama interpretativo. Dichas teorías tienen un origen sociohistórico, pero son incorporadas mediante procesos abstractivos en el sistema representacional de los individuos. En el próximo artículo, ofrecemos una investigación empírica sobre la estructura representacional de un grupo de teorías implícitas alternativas, relacionadas con el desarrollo y la educación.

\section{Resumen}

Las teorias del hombre de la calle se analizan utilizando una doble perspectiva, la de la psicologia social europea y la del procesamiento de información. La primera se centra en el origen social del conocimiento y analiza el carácter explicito - implicito de las teorias. Por su parte, la perspectiva cognitiva puede aportar conocimientos sobre las propiedades estructurales y funcionales de las teorias implicitas, asimilándolas a esquemas.

\section{Summary}

Layman theories are analysed, using two different approaches, the european social psychology and the information processing framenork. The former is focused on the social nature of cognition ands discusses the implicit or explicit character of the theories. The latter provides knowledge about the structural and functional properties of implicit theories, assimilating them to sibemas.

\section{Résumé}

Les théories de l'bomme de la rue s'analysent selon une double perspective: celle de la psychologie sociale européenne et celle du traitement de l'information. La première souligne l'origine social de la connaissance et analyse le caractère explicite ou implicite de ces théories. De son côté la perspective cognitive peut apporter de nouvelles connaissances sur les propriétés structurelles et fonctionnelles des theiories implicites en les assimilant à des scbemes.

\section{Referencias bibliográficas}

AbELSON, R. P.: “Concepts for representing mundane reality in plans». En D. G. Bobrow y A. Collins (Eds.): Representation and understanding. Studies in Cognitive Science. Academic Press, 1975.

ABEL.SON, R. P.: «Psychological status of the script concept». American Psychologist, 36, 7, págs. 71 9-729, 1981 .

Alion, L., y SEl.lgman, M. E. P.: «On the cognitive component of learned helplessness and depression. A critical analisis». En BOWER, G. H. (Ed.): The psychology of learning and motivation. Vol. 8. Academic Press, 1979.

ALIoY, L., y TAвACHNIK, N.: «Assessment of covariation by humans and animals: The joint influence of prior expectations and current situacional information». Psychological Review, 91, 1, págs. $112-149,1984$.

ANDERson, J. R., y Ross, B. H.: «Evidence against a semantic: Episodic distinction». J. of Exp. Psycbol.: Human Learn. and Mem., 6, 5, págs. 441-446, $19^{80}$.

BARTI.ETT, F. C.: "Remembering". A Study in Experimental and Social Psychology. Cambridge: Cambridge University Press, 1932.

BORGIDA, E., y NISBETT, R. E.: "The diferential impact of abstract vs. concrete information on decision". I. of Applied Social Psycbology, 7, págs. 258-271, 1977. 
BRomi.eY, D. B.: Personality Description in Ordinary Language. London. Wiley, 1977.

Bruner, J. S., y TAGiuri, R.: «The perception of people». En G. Lindzey (Ed.): Handbook of social psychology. Cambridge, Mass.; Addison; Wesley, 1954.

Chan, D., y JACKSON, D.: "Implicit theory of psychopatology". Multivariate Bebavioral Researcb, I4, págs. 3-19, 1979.

DE VEGA, M.: "Sobre el pensamiento cientifico». Revista de Investigación Psicológica. Universidad de La Laguna. Vol. 0,1983 .

De VegA, M.: Introducción a la Psicologia Cognitiva. Alianza Editorial, 1984.

Duval., S., y WICkI.und, R. A.: A tbeory of objective self-awareness. Nueva York: Academic Press, 1972.

ERicCSON, K., y Simion, H.: «Verbal reports as dats». Psychological Review, 87, 3, págs. 2 i s-2 51 , 1980.

FI.AveI.I., J. H., y Ross, L. D. (Eds.): New directions in the Study of social-cognitive development. Cambridge: Cambridge University Press, $198 \mathrm{r}$.

FI.AVEI.I. J. H., y WEI.I.MAN, H. M.: Metamemory. In perspectives on the development of memory and cognition. Kait, R. V. y Hagen, J. W. (Eds.). Hillsdale, N. J. Erlbaum, 1977.

Forgas, J. P.: Social Cognition: Perspectives on Everyday Understanding. Academic Press, 1981.

Garzón, A.: «La Psicologia Social Cognitiva». Boletín de Psicologia. Universidad de Valencia, 3, págs. 77-94, 1984 .

GoOdNow, J.: «Everyday ideas about cognitive development». En J. P. Forgas (Ed.): Social Cognition. Perspectives on Everyday understanding. Academic Press, $198 \mathrm{I}$.

Graesser, A. C.; Gordon, S. E., y SAWYER, J. D.: «Recognition memory for typical an atypical actions in scripted activities: Test of a script pointer-tag hypothesis». J. of Verb. Learn. and Verb. Behav., 18, págs. 319-332., 1979.

HeIDER, F.: The Psycbology of Interpersonal Relations. Nueva York, Wiley, 1958.

Higgins, E. T.; Herman, C. P., y Zanna, M. P.: Social Cognition. The Ontario Symposium, 1. Lawrence Erlbaum Associates, Hillsdale, Jersey, $198 \mathrm{I}$.

HONES, T.: "Children's implicit theories of their peers: a development analysis». British Journal of Psychology, 70, págs. 417-424, 1979.

Kahneman, D., y TVERSKY, A.: «Subjetive probability: A judgment of representativeness». Cognitive Psycbology, 3, págs. 430-454, 1972.

Kahneman, D.; Siovic, P., y TVERSKY, A. (Eds.): Judgment un der uncertainty: Heuristics and biases. Cambridge University Press. Cambridge, 1982.

Kahneman, D., y TVerSky, A.: "On the psychology of prediction». Psychological Review, 80, págs. 237-251, 1973.

KarmiLoff-Smith, A., e INHELDER, B.: «If you want to get ahead get a theorym. Cognition, 3, págs. 195-2 I 2, 1974. Trad. cast. en Infancia y Aprendizaje, 1981, 13, 69-88.

KinTSCH, W., y VAN Dijk, T. A.: «Toward a Model of Text Comprehension and Production». Psychological Review, 85,5 , págs. $363-394,1978$.

KHUN, T. S.: The Structure of scientific revolutions (2nd ed.). University of Chicago Press, Chicago, 1970.

KUIPERS, B. J.: "A frame frames». En Bobrow, D. G., y Col.Jins, A. (Eds.): Representation and Understanding studies in Cognitive Science. Academic Press, 1975.

LAmb, M. E., y Sherrod, L. R.: «Infant Social Cognition: Empirical and Theoretical Considerations». Lawrence Erlbaum Associates publisbers. Hillsdale, New Jersey, 1981.

LANGER, E.: «The Illusion of control». Journal of Personality and Social Psychology, 32, págs. 31 I -32 I, 1979.

LEPPER, M.; GREENE, D., y NISBETT, R.: «Undermining children's intrinsic interest with extrinsic rewards. Journal of Personality and Social Psycbology, 28, págs. 1 29-137, 1973.

MinSKY, M.: “Frame-system theory». En SHANK, R. C., y NASHWEBBER, B. L. (Eds.): Theoretical issues in Natural Language Processing. "Preprints of a conference at MIT", 1975.

NeISSER, U.: Cognition and Reality. Principles and Implications of Cognitive Psychology. Freeman and Company, 1976.

NisbetT, R. E.; KRANTZ, D. H.; JEPSON, C., y KundA, Z. P.: "The Use of Statistical Heuristics in Everyday Inductive Reasoning». Psycbological Review. Vol, 90, 4, 1983.

NisbetT, R., y Ross, L.: Human inference. Strategies and Sbortcomings of social judgement. Englewood Cliffs. New Jersey. Prentice Hall, 1980.

Peterson, C. R., y BeACH, L. R.: «Man as an intuitive statistician». Psycbological Bulletin, 68, págs. 29-46, 1967.

Piaget, J.: La répresentation du monde chez l'enfant. Alcan, París. Traducción al castellano en Espasa Calpe, Madrid, $1933,1926$.

- The Psychology of intelligence. Londres, Routledge and Kegan Paul. Traducido del original francés en Colin, París, 1947. Traducido al castellano en "Psique», Buenos Aires, 1959, 1950.

POLANSKY, M.: Knowing and being. University of Chicago Press, 1969.

RANDS M., y LEVINGER, G.: «Implicit Theories of relationship: An Intergenerational Study". Journal of Personality and Social Psychology, 37, 5, págs. 64, 661, 1979.

Rosenberg, S., y Sedi.AK, A.: "Structural representations of Implicit Personality Theory». En Advances in Experimental Social Psychology, 6. Berkowitz, L. (Ed.). Nueva York y Londres. Academic Press, I 972.

ROSENHART, D.: On being sane in insane places. Science. 179, págs. 250-258, 1973.

Ross, L.: "The intuitive psychologist and his shortcomings". En BERKOwITZ, L. (Ed.): Advances in Experimental Social Psycbology. Nueva York, Academic Press, 1977.

Ross, L.: "The intuitive scientist formulation and its developmental implications». En FI.AVEJ.I., J. H., y Ross, L. (Eds.): Social Cognitive development: frontiers and possible futures. Cambridge University Press, 1981 .

ROSS, L., y LEPPER, M. R.: "The perseverance of beliefs: Empirical and normative considerations». En SHWEDER, R. A. (Ed.): New directions for metbodology of social and bebavioral science: Fallible judgement in bebavioral research. Jossey-Bass, San Francisco, 1980.

Rorthbart, M.; Fulero, S.; Jensen, C.; HowARD, J., y Birrei.J., B.: «From individual to group impressions: Availability heuristics in stereotupe formation». Journal of Experimental Social Psychology, 14 , págs. 237-255, 1978. 
Rumeri.hART, D. E., y ORTONY, A.: "The representation of knowledge in memory". En ANDERSON, R. C.; SPIRO, R., y MONTAGUE, W. E. (Eds.): Schooling and the acquisition of knowledge. Lawrence Erlbaum Associates, Hillsdale, New Jersey, 1977.

Schank, R. C., y Abeison, R. P.: Scripts, Plans, Goals and understanding. An Inquiry into buman knowledge structures. Lawrence Erlbaum Associates. New Jersey, 1977.

SCHNEIDER, D. J.: «Implicit personality theory: A review». Psychological Bulletin, Vol. 79, págs. 294-305, 1973.

Seonane, J.: "Psicologia Cognitiva y Psicologia del Conocimiento». Boletin de Psicologia. Universidad de Valencia, págs. $2 \xi-44,1982$.

Shantz, C. U.: «The development of Social Cognition». En Hetherinciton, E. M. (Ed).: Review of Child development research, 5. University of Chicago Press. Chicago, 1975.

Schust ACK, M. W., y Stenberg, R. J.: «Evaluation of evidence in causal interference». J. of Exp. Psychol. Gener. Vol. 110, 1, págs. 101-120, 1981 .

SNyder, M. L., y WiCki.und, R. A.: "Attributional ambiguity". En Nev Directions in Attribution Research 3. HARveX, J. H.; ICKES, W. J., y KIDD, R. F. (Eds.). Nueva Jersey, Hillsdale, Erlbaum (en prensa).

SPIRO, R. J.: «Constructive Proceses in Prose comprehension and recall». En SPIR(), et al., (eds.): Theorical Issues in reading comprebension. Lawrence Erlbaum Associates, New Jersey, 1980.

TAYIOR, S. E., y FISKE, S. T.: "Salience, attention and attribution: Top of the head phenomena». En BERKOWITZ, L. (Ed.): Advances in experimental social psychology. Vol. I1, Nueva York, Academic Press, 1978.

Tweney, R.; Doherty, M., y MYNatt, C.: On scientific thinking. Nueva York, Columbia University Press, 1981 .

VAII.ARCHER, R. R.: An introduction to self theory. En "The self in social psychology". WEGNER, P. M., y VALl.ACHER, R. R. (Eds.). Nueva York, Oxford, 1980.

WEGNER, D.: «Attribute generality: The development and articulation of attributes in person perceptionm. $J$. of Research in person, 1977.

WEGNER, D. M., y VALLACHer, R. R.: Implicit Psycbology: An Introduction to Social Cognition. Nueva York, Oxford University Press, 1977.

Wegner, D. M., y VAliACHER, R. R.: «Common-sense psychology». En Forgas, J. P. (Ed.): Social Cognition. Perspectives on everyday Understanding. Academic Press, $198 \mathrm{t}$.

WHITE, P.: «Limitations on verbal reports of internal events: $A$ regulation of Nisbett and Willson and Wilson and of Bem». Psychological Review, 87, págs. 105-112, 1980.

WISH, M.; DeUTSCH, M., y KAPLAN, S.: «Perceived dimensions of interpersonal relations». J. of Pers. and Soc. Psych. Vol. 33, págs. 409-420, 1976. 